\title{
RELATO DE EXPERIÊNCIAS DO PROGRAMA DE EXTENSÃO DA UNIVERSIDADE DO EXTREMO SUL CATARINENSE ATRAVÉS DO “CURSO DE CULINÁRIA PROFISSIONAL E SEGURANÇA ALIMENTAR”
}

\author{
REPORT OF EXPERIENCES OF THE EXTREME SOUTH CATHARIAN UNIVERSITY \\ EXTENSION PROGRAM THROUGH THE "PROFESSIONAL CULINARY COURSE AND \\ FOOD SAFETY”
}

Tamara Justin da Silva ${ }^{1}$, Renata dos Santos Albino ${ }^{1}$, Fabiane Maciel Fabris ${ }^{1}$, Janete Trichês ${ }^{2}$

\begin{abstract}
RESUMO
O Projeto de Extensão "Curso de Culinária Profissional e Segurança Alimentar", tem como finalidade proporcionar o desenvolvimento de ações criando alternativas voltadas a adolescentes e jovens de baixo nível socioeconômico de integrarem à sociedade e ao mercado de trabalho, com uma adequada qualificação profissional. $O$ projeto foi realizado na Associação Beneficente ABADEUS, localizada no bairro Cristo Redentor - Criciúma, SC. Consiste na realização de aulas práticas e teóricas sobre técnicas de culinária e gastronomia, com ênfase também principalmente em segurança alimentar, visando incorporação de boas práticas na fabricação e higiene dos alimentos, conforme estabelece a ANVISA através da resolução RDC n. ${ }^{\circ}$ 216/2004.O trabalho trata-se de um relato de experiência, que tem por objetivo apresentar as atividades e experiências realizadas com as turmas participantes do projeto durante o período de 2018/1 e 2019/2. Este projeto mostrou-se fundamental para formação e aperfeiçoamento dos participantes, com resultados positivos no desempenho dos alunos, observamos melhor entendimento sobre nutrição, higiene e boas práticas na manipulação dos alimentos. O curso acarretou ainda desenvolvimento pessoal através de habilidades como trabalho em equipe, autonomia, liderança etc. Portanto, agregou oportunidades aos jovens participantes da comunidade Cristo Redentor visando assim melhores qualificações para o mercado de trabalho, além das práticas de extensão universitária que proporcionaram a troca de saberes entre as acadêmicas e os alunos.
\end{abstract}

Palavras-chave: ABADEUS; Curso de Culinária Profissional; Extensão Universitária; Segurança Alimentar e Nutricional.

\begin{abstract}
The Extension Project "Professional Cooking Course and Food Security", aims to provide the development of actions creating alternatives aimed at adolescents and young people of low socioeconomic level to integrate into society and the labor market, with an adequate professional qualification. The project was carried out at the Associação Beneficente ABADEUS, located in the Cristo Redentor neighborhood - Criciúma, SC. It consists of conducting practical and theoretical classes on cooking and gastronomy techniques, with an
\end{abstract}

\footnotetext{
${ }^{1}$ Universidade do Extremo Sul Catarinense - UNESC: Curso de Nutrição. E-mail: fabifabris@unesc.net

${ }^{2}$ Universidade do Extremo Sul Catarinense - UNESC: Curso de Direito
} 
RELATO DE EXPERIÊNCIAS DO PROGRAMA

DE EXTENSÃO DA UNIVERSIDADE DO EXTREMO SUL

CATARINENSE ATRAVÉS DO “CURSO DE CULINÁRIA

PROFISSIONAL E SEGURANÇA ALIMENTAR"

emphasis also mainly on food safety, aiming at incorporating good practices in food manufacturing and hygiene, as established by ANVISA through resolution RDC No. 216/2004. The work is an experience report, which aims to present the activities and experiences carried out with the classes participating in the project during the period of 2018/1 and 2019/2. This project proved to be fundamental for the training and improvement of the participants, with positive results in the students' performance, we observed a better understanding of nutrition, hygiene and good practices in food handling. The course also brought about personal development through skills such as teamwork, autonomy, leadership etc. Therefore, it added opportunities to the young participants of the Cristo Redentor community, thus seeking better qualifications for the job market, in addition to the university extension practices that provided the exchange of knowledge between academics and students.

Keywords: ABADEUS; Professional CookingCourse; University Extension; Food and nutritions ecurity.

\section{INTRODUÇÃO}

Segundo Garcia e Castro (2010) a culinária é um conjunto de regras relacionadas à alimentação, onde de acordo com o cardápio, temperos, técnicas de preparo, as formas de apresentar e servir, permitem identificar a culinária de uma região ou nação. Apresentar a alimentação na perspectiva da culinária, é justamente relacionar os aspectos culturais e valores simbólicos com a forma de se alimentar. Com isso, a culinária pode trazer uma importante contribuição para o campo da alimentação e nutrição sendo um objeto de estudo, já que considera a alimentação como um sistema organizado.

A inserção dos adolescentes e jovens no mercado de trabalho vem crescendo ao longo dos anos em um cenário de crescentes exigências profissionais. Cerca de 1/3 dos adolescentes com idade entre 15 e 17 anos e aproximadamente a dos jovens de 18 e 19 anos encontravam-se inseridos no mercado de trabalho em 2001 no Brasil, associando trabalho ou estudo ou apenas trabalhando. (SABÓIA; SOARES; KAPPEL, 2004).

As práticas de extensão universitária proporcionam importantes contribuições para a sociedade e para os acadêmicos.

As atividades de extensão universitária são uma forma de interação que deve ocorrer entre a universidade e a comunidade em que está inserida, atuando como uma ponte permanente entre o instituto de ensino superior e a sociedade. Como parte da Constituição Federal Brasileira de 1988, as atividades de extensão constituem uma possibilidade para as universidades exercerem sua autonomia didática, científica, administrativa, financeira e de gestão de ativos, seguindo o princípio da 
RELATO DE EXPERIÊNCIAS DO PROGRAMA

DE EXTENSÃO DA UNIVERSIDADE DO EXTREMO SUL

CATARINENSE ATRAVÉS DO “CURSO DE CULINÁRIA

PROFISSIONAL E SEGURANÇA ALIMENTAR"

indivisibilidade do ensino, pesquisa e extensão, que são os pilares das universidades. (SIQUEIRA et al. 2017).

Sendo assim, o objetivo do estudo, é relatar as atividades e experiências por meio da extensão universitária através da implementação do Curso de Culinária Profissional e Segurança Alimentar na instituição ABADEUS, apresentando a atuação acadêmica na comunidade, fortalecendo o conhecimento e aprimorando a qualidade profissional dos jovens participantes do projeto.

\section{PROCEDIMENTOS METODOLÓGICOS}

A realização do projeto foi feita através da parceria entre a Universidade do Extremo Sul Catarinense (UNESC) e a Associação Beneficente ABADEUS, que realizou a seleção e inscrição dos jovens participantes, que estavam inscritos no programa Jovem Aprendiz da instituição ABADEUS.Os métodos utilizados para a realização deste projeto envolveram profissionais de diversas áreas, que juntos buscam capacitar jovens de até dezoito anos de idade como manipuladores de alimentos para ingressarem no mercado de trabalho.

As aulas foram realizadas nas dependências da ABADEUS que juntamente com a UNESC, forneceram, para a realização das aulas, materiais como projetor multimídia (Datashow), televisão, acesso à internet, EPIs (jalecos), toucas, ingredientes para a elaboração das preparações e utensílios necessários em uma cozinha para a elaboração das preparações.As aulas práticas eram realizadas segundo disponibilidade de ingredientes, equipamentos e utensílios para preparação das receitas.

O curso foi realizado adotando-se uma configuração em que metade da aula foi de abordagem teórica e a outra metade prática. As aulas teóricas foram ministradas em sala de aula de forma expositiva e dialogada com a utilização do projetor multimídia e televisão de acordo com o conteúdo que seria apresentado. As aulas foram elaboradas utilizando-se como base a RDC n. ${ }^{\circ}$ 216/2004 e o Guia Alimentar Para a População Brasileira. Foram utilizadas também cartilhas sobre PANCs, documentos governamentais relacionados à alimentação, técnicas de preparo de alguns alimentos, branqueamento e congelamento, organização de geladeira, dentre outros materiais educativos. Todos os materiais passaram pela aprovação das professoras coordenadoras do projeto antes de cada aula, garantindo que a informação passada fosse verídica com embasamentos científicos. No que se refere a parte prática, tendo em vista 
RELATO DE EXPERIÊNCIAS DO PROGRAMA

DE EXTENSÃO DA UNIVERSIDADE DO EXTREMO SUL

CATARINENSE ATRAVÉS DO “CURSO DE CULINÁRIA

PROFISSIONAL E SEGURANÇA ALIMENTAR"

que a capacidade da cozinha comporta em média 15 pessoas, os alunos foram dividimos em dois grupos. O primeiro grupo, devidamente uniformizado com jalecos e toucas, elaborava as preparações e o segundo grupo era responsável pela organização e limpeza dos utensílios e da cozinha e pela apresentação dos pratos. A cada semana os grupos trocavam as funções.

Figura 1: Aula teórica com dinâmica realizada no segundo semestre de 2018

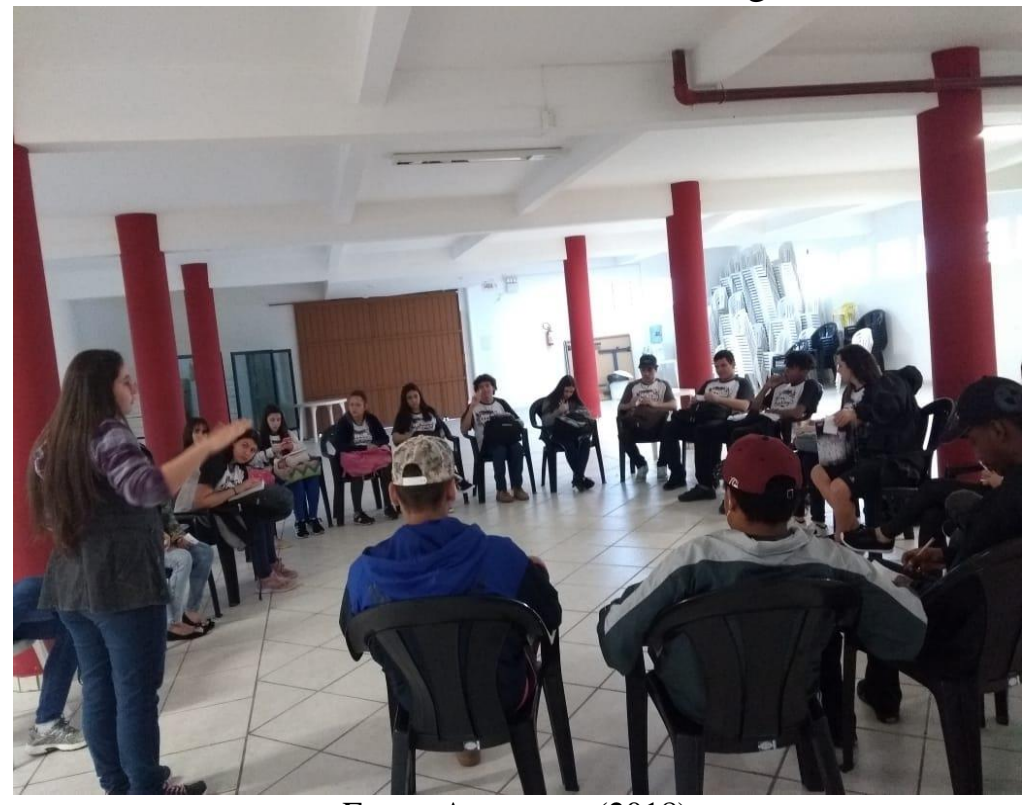

Fonte: As autoras (2018)

\section{DESENVOLVIMENTO}

Com as constantes transformações que vem ocorrendo nos hábitos alimentares de cada indivíduo, a alimentação fora do lar vem adquirindo força, que é subordinada por diversos motivos, como tipos de serviços oferecidos, despesa com alimentação, qualidade do serviço, segurança alimentar e consequente aumento da oferta de serviços relacionados a alimentação. As políticas públicas atuais de promoção da saúde reconhecem a importância da alimentação saudável nos diversos estágios da vida. Nesse contexto, busca-se através das aulas práticas e teóricas que serão ministradas fornecer conhecimento técnico e científico para a garantia da qualidade biológica, sanitária, nutricional e tecnológica dos alimentos, também o seu aproveitamento e a redução do seu desperdício.

Os jovens participantes do projeto estão cadastrados no Programa Jovem Aprendiz na instituição ABADEUS. "O programa Menor Aprendiz proporciona aos jovens oportunidades de ingressarem qualificados no mercado de trabalho e oferece incentivos para 
RELATO DE EXPERIÊNCIAS DO PROGRAMA

DE EXTENSÃO DA UNIVERSIDADE DO EXTREMO SUL

CATARINENSE ATRAVÉS DO “CURSO DE CULINÁRIA

PROFISSIONAL E SEGURANÇA ALIMENTAR”

as empresas como a redução de impostos." (PEREIRA, 2011, p.9). Além de agregar mais conhecimentos aos jovens e qualificá-los para o mercado de trabalho, uma pesquisa feita com 99 jovens relatou que a expectativa por fazer curso universitário apareceu para 74 aprendizes, sendo que 16 indicaram mais de uma opção de curso (FREITAS; OLIVEIRA, 2012).

O projeto iniciou-se no início do segundo semestre de 2018, contando com a participação de 22 jovens, e 21 no primeiro semestre do ano de 2019, totalizando 43 formandos que receberam certificados do Curso de Culinária Profissional e Segurança Alimentar. A carga horária total foi de 48 horas, com duração de 12 semanas.

Os encontros foram realizados uma vez por semana com carga horária de 04 horas e frequência controlada através de lista de chamada. Os temas relacionados aos encontros abordam assuntos como: alimentação saudável, adequada e sustentável; nutrição básica; técnicas gastronômicas; execução de cardápios; restrições alimentares (doença celíaca e intolerância a lactose); aproveitamento integral dos alimentos e desperdício; panificação e confeitaria; saladas; cereais e massas; laticínios e ovos, vegetarianismo; PANCs; e boas práticas de produção e manipulação de alimentos, de acordo com a RDC n. ${ }^{\circ}$ 216/2004 da ANVISA. Além disso, também foram realizadas fichas técnicas de algumas preparações, demonstrando o baixo custo das receitas realizadas.

Figura 2: Atividades realizadas durante o projeto

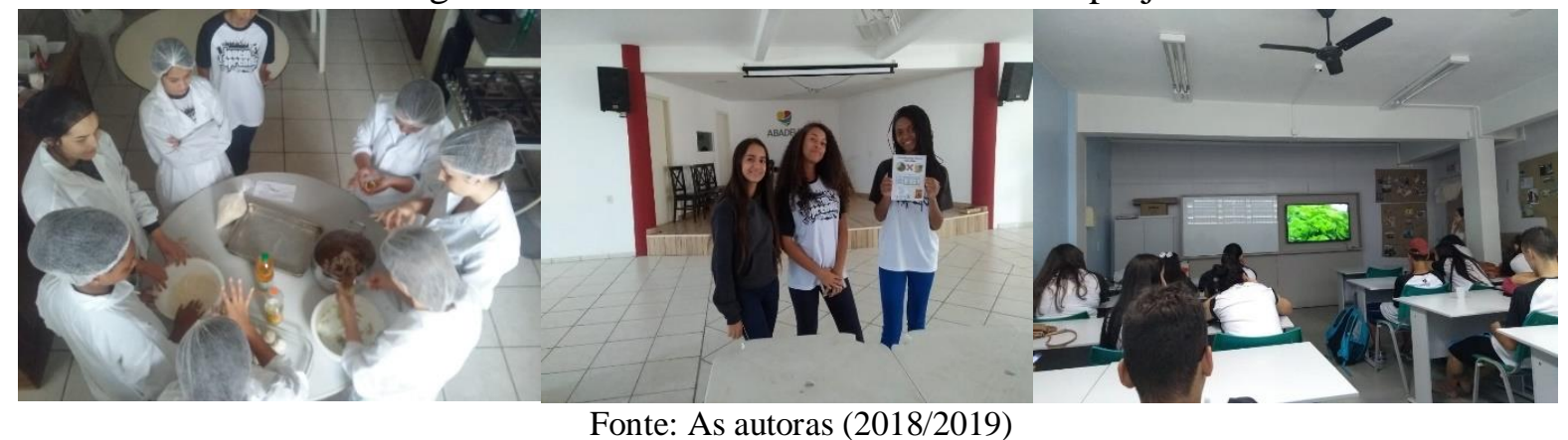

Os alunos do projeto demonstraram-se interessados sobre os assuntos abordados em aula, questionando, realizando as atividades propostas e opinando novas ideias de assuntos e receitas para as próximas aulas. Ambas as turmas demostraram uma melhora em seu rendimento na cozinha em diversos quesitos, tais como: organização, higiene, interpretação e elaboração das receitas, limpeza, boas práticas de manipulação etc. 
RELATO DE EXPERIÊNCIAS DO PROGRAMA

DE EXTENSÃO DA UNIVERSIDADE DO EXTREMO SUL

CATARINENSE ATRAVÉS DO “CURSO DE CULINÁRIA

PROFISSIONAL E SEGURANÇA ALIMENTAR"

Foi realizado com a turma de 2019/1, uma avaliação em dupla onde abordava assuntos referentes às aulas, para que se pudesse avaliar o conhecimento dos alunos. Essa avaliação foi realizada em dois momentos, no início do curso, quando eles ainda não haviam recebido nenhum tipo de informação sobre os assuntos das acadêmicas, e no final do curso, sendo aplicada na última aula.

Tabela 1: Resultados das avaliações aplicadas durante o projeto.

\begin{tabular}{|c|c|}
\hline \multicolumn{2}{|c|}{ Avaliação Inicial } \\
\hline Notas acima da média 6 & 2 \\
\hline Notas com a média 6 & 2 \\
\hline Notas abaixo da média 6 & 3 \\
\hline \multicolumn{2}{|c|}{ Avaliação Final } \\
\hline Notas acima da média 6 & 5 \\
\hline Notas com a média 6 & 1 \\
\hline Notas abaixo da média 6 & 1 \\
\hline
\end{tabular}

Fonte: As autoras (2019)

Os resultados que foram obtidos durante as avaliações (tabela 1) mostra a evolução que os jovens participantes do projeto tiveram. Durante a primeira avalição, apenas 2 duplas de alunos alcançaram nota acima da média, sendo a maior delas a nota 7 , enquanto que na segunda avaliação (contendo as mesmas questões, porém com os alunos já tendo o conhecimento dos assuntos ministrados nas aulas), 5 duplas conseguiram notas acima da média, sendo a maior delas a nota 9. 
Figura 3: Encerramento das turmas de 2018/2 e 2019/1, respectivamente.

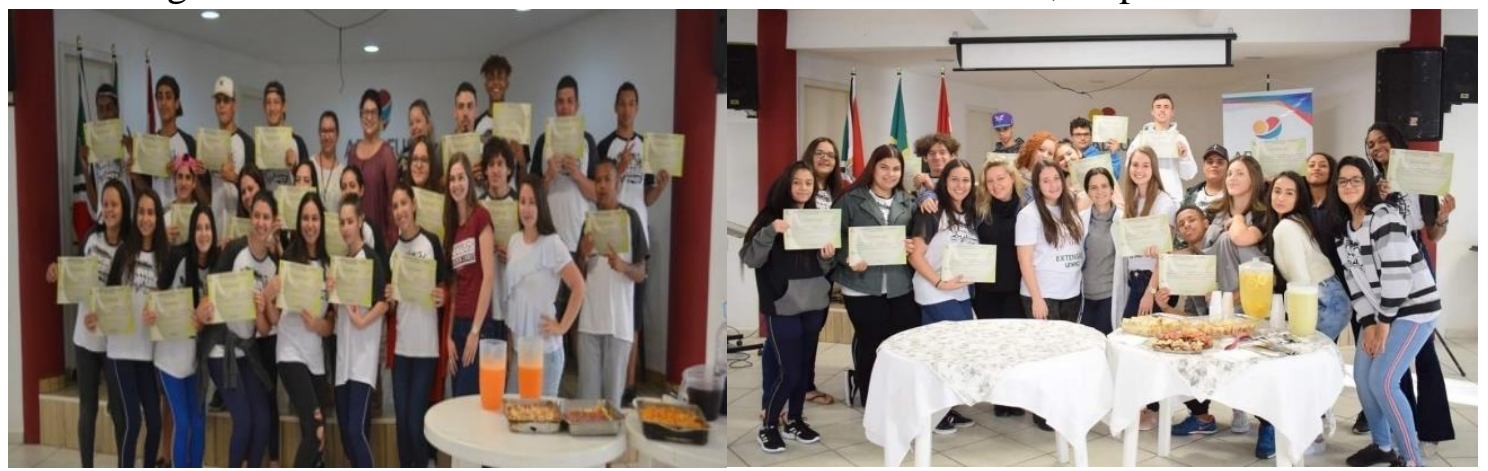

Fonte: As autoras (2018/2019)

\section{CONSIDERAÇÕES FINAIS}

A extensão universitária proporciona importantes contribuições para a sociedade e para os acadêmicos. A partir do momento em que é colocado em prática com a comunidade aquilo que se aprende em sala de aula, ambos os lados se beneficiam com a troca de conhecimento e experiências.

É válido ressaltar que,

A relação da universidade com a comunidade se fortalece pela Extensão Universitária, ao proporcionar diálogo entre as partes e a possibilidade de desenvolver ações sócio-educativas que priorizam a superação das condições de desigualdade e exclusão ainda existentes. E, na medida em que socializa e disponibiliza seu conhecimento, tem a oportunidade de exercer e efetivar o compromisso com a melhoria da qualidade de vida dos cidadãos. (ROCHA, 2007 apud RODRIGUES et al. 2013)

As práticas de extensão proporcionam um saber diferenciado, focado para a sociedade. Devido a isso, as práticas de extensão se mostraram eficientes em relação ao projeto, onde o mesmo permitiu a ampliação do conhecimento dos jovens através das aulas teóricas e práticas. Com isso, os jovens ganharam um diferencial no mercado de trabalho, por possuírem o certificado do curso e o aprendizado em relação ao ambiente de trabalho, higiene, preparação de alimentos etc. Além disso, permitiu o ensino sobre uma alimentação e preparações saudáveis, aproveitamento integral de alimentos, restrições alimentares etc. Assuntos que estão presentes durante o cotidiano, tanto no trabalho quanto no ambiente familiar, e que possam estar colocando em prática, iniciando um ciclo de troca de conhecimentos entre acadêmicos e comunidade. 


\section{FONTES DE FINANCIAMENTO}

Fonte de Financiamento: UNESC.

\section{REFERÊNCIAS}

CONSELHO NACIONAL DE SEGURANÇA ALIMENTAR E NUTRICIONAL (CONSEA). Lei de segurança alimentar e nutricional: Conceitos - Lei $n^{\circ} 11.346,15$ de setembro de 2006. Disponível em:

<http://www.epsjv.fiocruz.br/sites/default/files/documentos/pagina/lei_11346-06.pdf $>$ Acesso em: 03 nov. 2019.

FREITAS, Maria de Fatima Quintas de; OLIVEIRA, Lygia Maria Portugal de. Juventude e Educação Profissionalizante: Dimensões Psicossociais do Programa Jovem Aprendiz.

Psicologia em Pesquisa, Paraná, v. 6, n. 2, p. 111-120, dez. 2012. Disponível em: $<$ http://pepsic.bvsalud.org/pdf/psipesq/v6n2/v6n2a04.pdf> Acesso em: 05 nov. 2019.

GARCIA, Rosa Wanda Diez; CASTRO, Inês Rugani Ribeiro. A culinária como objeto de estudo e de intervenção no campo da Alimentação e Nutrição. Ciência \& Saúde Coletiva, São Paulo, v. 16, n. 1, p. 91-98, 2011. Disponível

em: $\langle$ http://www.scielo.br/scielo.php?script=sci arttext\&pid=S1413-81232011000100013> Acesso em: 04 nov. 2019.

PEREIRA, Joelma Maria. O Aprendiz no mercado de trabalho. Facider, Mato Grosso, n.4, p. 1-15, 2011. Disponível em:

<http://www.seicesucol.edu.br/revista/index.php/facider/article/view/39/91> Acesso em: 04 nov. 2019.

SABOIA, A. L.; SOARES, B. C.; KAPPEL, D. B. Adolescentes e jovens no brasil: escolarização e inserção no mercado de trabalho. In: XIV Encontro Nacional de Estudos Populacionais, 2010, Caxambú. Anais..., Caxambú: ABEP, 2010. Disponível em: <http://www.abep.org.br/ abeporgb/publicacoes/index.php/anais/article/viewFile/1395/13 60> Acesso em: 05 nov. 2019.

SIQUEIRA, Samylla Maira Costa et al.Atividades de extensão, promoção da saúde e desenvolvimento sustentável: a experiência de um grupo de pesquisa em enfermagem. EEAN, Bahia, p. 1-7, fev. 2017. Disponível em:

$<$ http://www.scielo.br/scielo.php?pid=S1414-

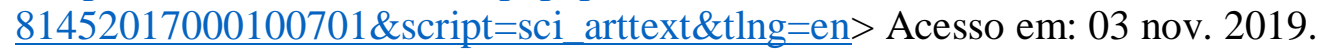

